\title{
COMPARAÇÃO DE PROPOSTAS PARA ALOCAÇÃO DOS CUSTOS DE PERDAS NA TRANSMISSÃO
}

\author{
Delberis Araujo Lima \\ UNESP - Campus de Ilha Solteira \\ Av. Barsil 56, Ilha Solteira - SP \\ Fone: (18) 37431150 \\ delberis@aluno.feis.unesp.br
}

\author{
Antonio Padilha-Feltrin \\ UNESP - Campus de Ilha Solteira \\ Av. Barsil 56, Ilha Solteira - SP \\ Fone: (18) 37431169 \\ padilha@dee.feis.unesp.br
}

\begin{abstract}
An analysis of the principal methods of transmission loss allocation acting for generators and loads is presented. Two methods based on the pro-rata technique, three marginal procedures, one method based on proportional sharing and one more method based on the theory of electric circuits are discussed. The algorithms are tested, firstly, with didactic systems for easy verification of potentialities and disadvantages, following of a result evaluations with a large systems (IEEE 118). The presented and discussed results consider: the location and the magnitude of generators and loads; the possibility of having agents well or badly located in each configuration of the net; the performance when variations in the network happen; and the discriminatory behavior. Conclusions and recommendations are stated.
\end{abstract}

KEYWORDS: Loss Allocation, Electricity Market, Transmission Losses.

\section{RESUMO}

Uma análise de desempenho dos principais métodos de alocação de perdas para geradores e cargas é realizada. São discutidos dois métodos baseados na técnica pro-rata, três procedimentos baseados em técnica incremental, um método baseado em participação proporcional e mais um método baseado na teoria de circuitos elétricos. Os

Artigo Submetido em 11/02/03

1a. Revisão em 19/04/04;

2a. Revisão em 14/09/04

Aceito sob recomendação do Ed. Assoc. Prof. Glauco Taranto algoritmos são testados, primeiramente, com exemplos didáticos de fácil verificação de potencialidades e desvantagens, para em seguida avaliar resultados com sistemas de porte maior (IEEE 118). Os resultados apresentados e discutidos verificam: a localização e a magnitude de geradores e cargas; a possibilidade de se ter agentes bem ou mal localizados em cada configuração da rede; o desempenho com alterações na rede elétrica; e o comportamento discriminatório. Conclusões e recomendações são apresentadas.

PALAVRAS-ChAVE: Alocação de Perdas, Mercado de Energia Perdas na Transmissão.

\section{INTRODUÇÃO}

Atualmente as empresas de transmissão de energia elétrica estão passando por significativas mudanças em seu novo ambiente econômico. Vários desafios têm surgido: como a determinação das capacidades ótimas de transferência com alocação de custos de congestionamento; e a determinação da alocação das perdas elétricas para geradores e cargas. Este artigo trata da determinação da alocação de perdas para geradores e cargas a partir dos métodos com mais destaque em aplicações práticas e dos com maiores potencialidades futuras.

Considerando que as perdas elétricas dependem das injeções de potências nas barras e são funções não lineares, é interessante realizar a análise da alocação das perdas a partir de mais de um procedimento. Deve-se destacar que a alocação dos custos das perdas para barras não afeta diretamente o funcionamento dos sistemas elétricos existentes, uma vez que o cálculo destes custos é realizado depois do despacho de cargas. Obviamente o tamanho e localização de geradores e cargas contribuem, para mais ou para menos, com as perdas elétricas. Este fato pode ser determinante na definição, por exemplo, de locais mais 
adequados para a conexão de novos geradores no sistema, e neste sentido a alocação das perdas pode ser um fator decisivo na expansão dos sistemas de geração.

Os métodos de alocação de perdas tem como objetivo distribuir entre geradores e cargas a responsabilidade por perdas na transmissão de forma equilibrada. Para atingir este equilibrio algumas características são importantes (Conejo et alii, 2001), como por exemplo: levar em conta a injeção de potência em cada barra; considerar a relativa localização da barra na rede de transmissão; ser consistente com a solução do fluxo de potência; ser estável diante de alterações pouco significativas de parâmetros da rede; fornecer efetivos incentivos ou punições para geradores e cargas considerando a sua relativa localização e magnitude na rede; e ser de simples compreensão e de fácil implementação.

Este artigo é direcionado para apresentar uma análise de desempenho dos principais métodos de alocação de perdas para geradores e cargas mostrando, primeiramente, resultados com exemplos didáticos de fácil verificação de potencialidades e desvantagens, para em seguida avaliar resultados com sistemas de maior porte. Os resultados apresentados e discutidos no artigo consideram a localização e a magnitude de geradores e cargas, e a possibilidade de se ter agentes bem ou mal localizados em cada configuração da rede. Também são feitos testes para verificar a sensibilidade dos métodos com alterações na rede elétrica. Com isto pretende-se fornecer uma contribuição no sentido de apresentar problemas e soluções para uma boa avaliação e aplicação de métodos de alocação de perdas. Os métodos discutidos são:

- Z-bus - método baseado na teoria de circuitos, utilizando a matriz Z-bus;

- $\quad$ PS - método de participação proporcional;

- ITL - método incremental de alocação;

- ITLPOS - método incremental de alocação sem alocação negativa (ITLPOS);

- AIL - alocação incremental de perdas. Este método é baseado nos fatores incrementais de perdas, com uma eficiente consideração da barra de referência;

- P - método usando a técnica Pro-Rata, com base na potência ativa; e

- I - método usando a técnica Pro-Rata, com base na corrente.

Uma revisão dos principais métodos para alocação de perdas pode ser encontrada em Fernandes et alii (2002), e uma análise comparativa é realizada em Conejo et alii
(2002). Métodos desenvolvidos para levar em conta somente contratos bilaterais, como o apresentado em Expósito et alii (2000) estão fora da análise deste artigo. A importância da análise de perdas elétricas ocorre em função da estimativa de seu custo anual ser da ordem de 1 (um) bilhão de reais (dados de 2001) no Sistema Brasileiro (Leite da Silva et alii, 2001).

\section{MÉTODOS DE ALOCAÇÃO DE PERDAS}

Diferentes propostas de alocação de perdas em redes elétricas surgiram nos últimos anos, tanto para transmissão quanto para distribuição. Obviamente o foco maior tem sido a transmissão devido aos valores atingidos e a grande quantidade de agentes com livre acesso às redes de transmissão. Nesta seção faz-se uma breve descrição dos métodos de alocação de perdas que serão discutidos.

\subsection{Método Baseado na Teoria de Circuitos}

O método empregado aqui é o Z-bus (Conejo et alii, 2001) que apresenta uma característica diferente e interessante que é o fato de explorar as equações de circuitos elétricos sem nenhuma simplificação. A idéia do método é distribuir as perdas do sistema (Ploss) entre as "n” barras do sistema, a partir da solução do fluxo de potência:

$$
P_{\text {loss }}=\sum_{k=1}^{n} L_{k}
$$

O componente de perdas $\left(L_{k}\right)$ é a fração de perdas do sistema atribuídas à barra "k". A obtenção do termo $\left(L_{k}\right)$ mostrado em (1) é feita a partir da matriz de admitância $(Y=G+j B)$ e o vetor complexo de tensão $(V)$ ou a partir da matriz de impedância $(Z=R+j X)$ e o vetor complexo de injeção de corrente (I). Partindo da equação de perdas temos:

$$
P_{\text {loss }}=\mathfrak{R}\left\{\sum_{k=1}^{n} V_{k} I_{k}^{*}\right\}
$$

Em que $\mathfrak{R}$ indica que se trata da parte real do vetor obtido do somatório. A referência Conejo et alii (2001) mostra que a componente de perdas associadas com a barra " $k$ " pode ser expressa por:

$$
L_{k}=\mathfrak{R}\left\{I_{k}^{*}\left(\sum_{j=1}^{n} R_{k j} I_{j}\right)\right\}
$$


O elemento $R_{k j}$ representa a parte real da matriz Z-bus que reflete a ligação entre a barra $\mathrm{k}$ e todas as outras barras do sistema.

O componente de perdas, $\left(L_{k}\right)$, considera os “ $n$ ” termos que representam o acoplamento entre as injeções de correntes de todas as " $n$ ” barras com a injeção de corrente na barra "k".

\subsection{Métodos de Alocação de Perdas Utilizando Procedimentos Incrementais}

Os procedimentos de alocação baseados no princípio de incrementos alocam as perdas para geradores e cargas a partir de fatores denominados como ITL e ITLPOS (Elgerd 1982; Galiana et alii., 2002; Schweppe et alii, 1988). Os fatores ITL's são obtidos a partir de uma solução do fluxo de potência. $O$ fator ITL de uma dada barra provém da mudança das perdas totais produzidas através da variação incremental de injeção de potência em cada barra. Deve ser destacado que o uso de fatores ITL's pode alocar perdas negativas para geradores e cargas, e isto pode ser interpretado como subsídio.

Quando não se deseja realizar alocação de valores negativos, como os realizados com os fatores ITL's, podese modificar os fatores ITL's obtendo-se os ITLPOS (Conejo et alii, 2002).

Recentemente, uma força tarefa coordenada pelo MAE (Mercado Atacadista de Energia), atualmente CCEE (Câmara de Comercialização de Energia Elétrica), elaborou uma metodologia para aplicação no sistema brasileiro. Este método é basedo nos fatores ITL, com uma forma eficiente para tratar a barra de referência (Leite da Silva et alii, 2001; Leite da Silva et alii, 2003), e nete trabalho é denominado de Alocação Incremental de Perdas (AIL).

\subsection{Método de Alocação de Perdas Utilizando Divisão Proporcional}

A proposta do método de alocação de perdas baseado no princípio da divisão proporcional (chamado de PS) é desenvolvida considerando a topologia da rede, que é em geral o objetivo do problema do transporte, em que a alocação de perdas é feita baseada na distribuição dos fluxos na rede como descrito em Bialek (1996).

A Fig.1 ilustra a idéia do princípio da divisão proporcional onde quatro linhas estão conectadas ao nó i, duas com fluxos entrando no nó i e duas com sentido dos fluxos saindo do nó i. O total do fluxo de potência que atravessa o nó i é Pi=100MW (40+60) dos quais 40\% provém do nó j e $60 \%$ provém do nó k.

Considerando 70 MW fluindo do nó i para o nó m pode-se obter, através do princípio da divisão proporcional, a potência de 42 MW (70*60/100) como sendo o valor que fluiu pela linha k-i. Similarmente o fluxo de $30 \mathrm{MW}$ entre il tem a potência de $18 \mathrm{MW}(30 * 60 / 100)$ que circulou na linha k-i.

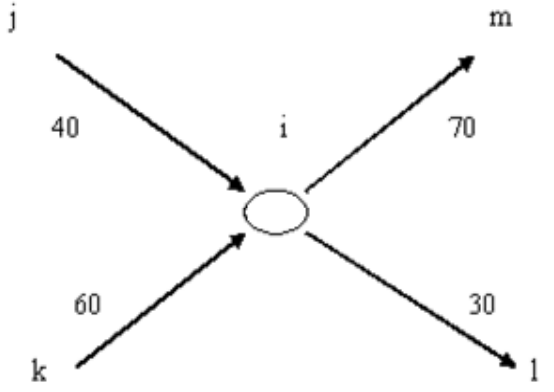

Figura 1. Princípio da divisão proporcional.

\subsection{Métodos Pro-Rata}

Primeiramente as perdas são divididas para geradores e cargas, $50 \%$ para cada, e então é feita uma alocação proporcional dentro de cada categoria. Assim uma barra com carga terá uma alocação de perdas correspondente a porcentagem de consumo de energia total (carga total conectada na rede). $\mathrm{O}$ mesmo princípio é aplicado às barras de geração. Um exemplo de aplicação é mostrado em Gonzalez and Bassagoiti (1999). A divisão das perdas pode ser realizada baseada nas injeções de potência ativa nas barras (denominado como P) ou nas injeções de corrente (chamado de I).

\section{TESTES, RESULTADOS E DISCUSSÕES}

Uma análise sobre o desempenho de métodos de alocação de perdas considerando-se diferentes situações e obtendo-se os resultados com mais de um método é realizada. Todos os métodos testados alocam perdas igual a zero para barras que não possuem nem geradores e nem cargas. Os métodos P, I, PS e ITLPOS sempre alocam uma parte de perdas para barras com geradores e cargas, e isto significa que todos os geradores e todas as cargas devem "pagar" por perdas elétricas. O ITL, AIL e Z-bus fazem alocação positiva e negativa de perdas, significando que geradores e cargas podem “pagar” (alocação positiva) ou “receber” (alocação negativa) por perdas elétricas.

Quando acontece alocação negativa para uma determinada barra, significa que esta barra deve receber incentivos 
monetários, e obviamente outras barras do sistema deverão contribuir a fim de pagar estes incentivos. Esta questão de "receber incentivos por perdas" é um tanto complexa e muitas vezes de difícil aceitação, pois as demais barras deverão pagar pelo total das perdas somado a estes incentivos. Realizar uma análise que separe barras que podem receber incentivos (chamadas de bem posicionadas na rede) de outras que devem pagar (denominadas mal posicionadas) pode ajudar a esclarecer algumas questões relativas ao incentivo. A princípio geradores instalados em centros de carga são bem posicionados e geradores distantes dos centros consumidores são mal posicionados. Cargas próximas de grandes centros geradores são bem posicionadas e distantes são mal posicionadas. Um dos objetivos deste trabalho é verificar e analisar a questão de incentivos monetários para geradores e cargas bem posicionadas, e verificar alocação discriminatória, respondendo algumas perguntas: (a) quais métodos produzem resultados discriminatórios?; (b)quais métodos levam barras bem posicionadas a pagar menos ou mesmo receber?; (c) pode-se utilizar o conceito de barras bem posicionadas para incentivar a instalação de geradores nestas barras em projetos de expansão?; (d) as barras que receberam acréscimo de geração em uma primeira expansão continuam bem posicionadas em uma segunda expansão?; (e) a instalação de outros agentes (geradores ou cargas) alteram o bom posicionamento anterior?; e (f) pequenas alterações na rede (tap de transformadores, capacitores, etc.) provocam alterações bruscas na alocação de perdas?

As respostas para estas questões e outras análises serão apresentadas usando-se os métodos discutidos anteriormente aplicados em três sistemas de energia elétrica:

- IEEE14 -com 14 barras, 20 linhas, 2 geradores e 13 cargas (Conejo et al., 2001), mostrado no Apêndice A;

- REDE5 - com 5 barras, 2 transformadores, 3 linhas. 2 geradores e 1 cargas (Glover and Sarma, 1987), mostrado no Apêndice B;

- IEEE118 - com 118 barras, 186 linhas, 17 geradores e 110 cargas (The Standard IEEE 118 test data (2003)), mostrado no Apêndice C.

As tabelas 1, 2 e 3 mostram os resultados obtidos para o sistema IEEE-14 barras padrão (caso base) e modificado acrescentando um gerador de $50 \mathrm{MW}$ e de $100 \mathrm{MW}$ na barra 8, respectivamente. Nas tabelas \$ é o custo das perdas alocado para cada barra, e é obtido como: \$=Lx50 (unidades da moeda empregada). Este procedimento foi adotado aqui para facilitar a comparação com a referência Conejo et alii (2001). Muitas vezes a alocação das perdas é apresentada após atribuição de uma porcentagem das perdas para geradores e cargas, por exemplo, 50\% para cargas e
50\% para geradores, como em Leite da Silva et alii (2001). Neste trabalho não é adotado este procedimento, porém destaca-se que não se tem dificuldades em aplicá-lo com os métodos discutidos aqui.

Através dos resultados obtidos na tabela 1, pode-se observar que todos os métodos de alocação de perdas atribuem o maior valor de perdas para a barra 1 (que possui o maior gerador do sistema), da mesma forma a barra 3 (possuidora da maior carga) recebe o maior valor de alocação de perdas entre as cargas.

A tabela 2 apresenta resultados com a inclusão de um gerador de $50 \mathrm{MW}$ na barra 8. As cargas e os outros parâmetros do sistema se mantiveram os mesmos do caso base. O objetivo é verificar o caso em que geradores e cargas estão melhores distribuídos no sistema. A mudança de geração da barra 1 para a barra 8 , verificada na tabela 2 , provocou uma diminuição significativa no total de perdas no sistema, em relação ao caso base. Para o caso base o custo total das perdas soma 677,7 (\$) e para o caso modificado apenas 448,5 (\$). Essa variação é devido a posição do gerador na barra 8 no sistema. O método Z-bus e o método AIL foram os métodos que apresentaram a maior sensibilidade com relação a essa variação, atribuindo um valor negativo de alocação de perdas para a barra 8 pelo benefício trazido ao sistema com a inserção do gerador. Deve-se notar que:

- A alocação com o Z-bus e o AIL mostra que a barra 8 recebe incentivos;

- Mesmo com o incentivo para a barra 8, o método Z-bus alocou um valor menor (em relação a tabela 2) para todas as barras. Isto mostra um procedimento não discriminatório, pois as perdas foram reduzidas e todas as barras obtiveram ganhos. 
Tabela 1. Custos alocados das perdas no sistema IEEE 14 para o caso base

\begin{tabular}{|c|c|c|c|c|c|c|c|}
\hline \multirow{3}{*}{$\begin{array}{c}\text { Barra } \\
n^{\circ}\end{array}$} & \multicolumn{2}{|c|}{ PRO-RATA } & \multirow{3}{*}{$\begin{array}{l}\text { PS } \\
\text { (\$) }\end{array}$} & \multirow{3}{*}{$\begin{array}{l}\text { ITL } \\
(\$)\end{array}$} & \multirow{3}{*}{$\begin{array}{c}\text { ITL(POS) } \\
(\$)\end{array}$} & \multirow{3}{*}{$\begin{array}{l}\text { AIL } \\
(\$)\end{array}$} & \multirow{3}{*}{$\begin{array}{l}\text { Z-bus } \\
\text { (\$) }\end{array}$} \\
\hline & $(\mathrm{P})$ & (I) & & & & & \\
\hline & (\$) & $(\$)$ & & & & & \\
\hline 1 & 322 & 274 & 324 & 309 & 339 & 335 & 382 \\
\hline 2 & 25 & 32 & 15 & 42 & 0 & 2 & 8 \\
\hline 3 & 130 & 116 & 144 & 147 & 44 & 164 & 139 \\
\hline 4 & 66 & 58 & 63 & 60 & 30 & 57 & 42 \\
\hline 5 & 10 & 9 & 8 & 8 & 21 & 6 & 4 \\
\hline 6 & 16 & 51 & 12 & 12 & 20 & 9 & 24 \\
\hline 7 & 0 & 0 & 0 & 0 & 0 & 0 & 0 \\
\hline 8 & 0 & 33 & 0 & 0 & 28 & 0 & 1 \\
\hline 9 & 41 & 41 & 39 & 36 & 29 & 36 & 28 \\
\hline 10 & 12 & 13 & 14 & 12 & 31 & 12 & 9 \\
\hline 11 & 5 & 5 & 5 & 4 & 28 & 4 & 3 \\
\hline 12 & 8 & 7 & 8 & 8 & 30 & 7 & 5 \\
\hline 13 & 19 & 18 & 19 & 18 & 33 & 18 & 13 \\
\hline 14 & 21 & 19 & 26 & 23 & 44 & 27 & 22 \\
\hline
\end{tabular}

Tabela 2. Custos alocados das perdas no sistema IEEE 14 para o caso modificado (com 50 MW na barra 8).

\begin{tabular}{cccccccc}
\hline Barra & \multicolumn{2}{|c}{ PRO-RATA } & & & & & \\
& $(\mathrm{P})$ & $(\mathrm{I})$ & PS & ITL & ITL(POS) & AIL & Z-bus \\
$\mathrm{n}^{\circ}$ & $(\$)$ & $(\$)$ & $(\$)$ & $(\$)$ & $(\$)$ & $(\$)$ & $(\$)$ \\
1 & 165 & 149 & 204 & 190 & 157 & 240 & 228 \\
2 & 17 & 16 & 13 & 29 & 68 & 5 & 6 \\
3 & 87 & 82 & 121 & 110 & 34 & 132 & 131 \\
4 & 44 & 41 & 44 & 35 & 18 & 30 & 27 \\
5 & 7 & 7 & 6 & 5 & 13 & 3 & 2 \\
6 & 10 & 35 & 9 & 8 & 16 & 5 & 22 \\
7 & 0 & 0 & 0 & 0 & 0 & 0 & 0 \\
8 & 46 & 46 & 7 & 10 & 15 & -28 & -24 \\
9 & 27 & 29 & 4 & 19 & 15 & 16 & 14 \\
10 & 8 & 9 & 2 & 7 & 18 & 6 & 6 \\
11 & 3 & 3 & 6 & 3 & 18 & 2 & 2 \\
12 & 6 & 5 & 6 & 5 & 24 & 6 & 5 \\
13 & 13 & 12 & 15 & 13 & 25 & 13 & 12 \\
14 & 14 & 14 & 11 & 16 & 30 & 19 & 18 \\
\hline
\end{tabular}

Tabela 3. Custos alocados das perdas no sistema IEEE 14 para o caso modificado (com $100 \mathrm{MW}$ na barra 8).

\begin{tabular}{cccccccc|c}
\hline Barra & \multicolumn{2}{l}{ PRO-RATA } & & & & & \\
& $(\mathrm{P})$ & $(\mathrm{I})$ & PS & ITL & ITL(POS) & AIL & Z-bus \\
$\mathrm{n}^{\circ}$ & $(\$)$ & $(\$)$ & $(\$)$ & $(\$)$ & $(\$)$ & $(\$)$ & $(\$)$ \\
1 & 80 & 72 & 111 & 102 & 120 & 144 & 116 \\
2 & 12 & 11 & 11 & 22 & 34 & 6 & 4 \\
3 & 60 & 57 & 92 & 89 & 30 & 117 & 124 \\
4 & 31 & 28 & 17 & 18 & 10 & 9 & 13 \\
5 & 5 & 5 & 4 & 3 & 7 & 1 & 1 \\
6 & 7 & 25 & 8 & 6 & 13 & 4 & 23 \\
7 & 0 & 0 & 0 & 0 & 0 & 0 & 0 \\
8 & 64 & 59 & 32 & 33 & 5 & -4 & -9 \\
9 & 19 & 20 & 0 & 7 & 4 & -1 & 3 \\
10 & 6 & 6 & 1 & 3 & 8 & 2 & 3 \\
11 & 2 & 2 & 2 & 2 & 12 & 1 & 1 \\
12 & 4 & 4 & 6 & 4 & 21 & 5 & 5 \\
13 & 9 & 9 & 16 & 10 & 21 & 11 & 11 \\
14 & 10 & 9 & 8 & 11 & 22 & 13 & 15 \\
\hline
\end{tabular}

Este incentivo monetário para a barra 8 (tabelas 2 e 3), atribuído pelo Z-bus e AIL, não é verificado pelos outros métodos. Os métodos P, I, PS e ITL aumentaram a atribuição de custo de perdas para a barra 8, e o ITLPOS alocou custo menor para a barra 8. De uma maneira geral a alocação para cada uma das barras diminuiu. A alocação de custo realizada com os métodos P, I, PS, AIL e ITL pode ser considerada discriminatória, pois a diminuição de perdas trouxe benefícios para todas as demais barras, exceto para a barra 8 (questão (a)). Este é um caso particular, porém didático, que mostra como alguns métodos podem fazer alocações que não consideram o posicionamento de geradores na rede. Para este caso o método Z-bus apresenta uma solução com incentivos monetários para a barra 8 sem penalizar outras. Este fato pode indicar que a barra 8 é bem posicionada na rede elétrica (questões (b) e (c)).

Para verificar o desempenho dos métodos em uma segunda expansão acrescenta-se um gerador de $50 \mathrm{MW}$ na barra 8, totalizando agora 100MW (tabela 3). Para a alocação mostrada na tabela 3 , o custo total de perdas diminuiu ainda mais e atingiu o valor de 308,0 (\$). Então, comparando com a tabela 2, observa-se que apenas o método ITLPOS registrou uma diminuição da alocação para a barra 8 . O Zbus e o AIL continuam indicando um valor negativo, porém agora o incentivo monetário é menor, quando esperava-se um incentivo maior pois o custo total de perdas diminuiu. 
Este fato indica que o uso do métodos Z-bus e AIL podem produzir resultados discriminatórios também, pois deve levar em conta o posicionamento e a potência gerada, e uma geração de $100 \mathrm{MW}$ em 8 é apontada como sendo pior que $50 \mathrm{MW}$, porém as perdas totais nas linhas do sistema indicam o contrário. O método Z-bus indica, na tabela 2, um gerador bem posicionado na barra 8, porém a instalação de um novo gerador na barra 8 (resultados da tabela 3), mostra que os incentivos que o gerador recebia foram diminuídos. Este fato indica que geradores bem posicionadas em uma condição de operação, podem deixar esta situação quando outros geradores são instalados (questão (d)). Estes resultados podem, a primeira vista, ser considerados discriminatórios, pois segundo Conejo et alii (2001) os métodos devem refletir as variações provocadas pelos agentes do sistema. Porém este comportamento é gerado pela alteração dos fluxos na rede que leva ao resultado obtido, e portanto esta alteração de fluxos deve tmabém ser considerada na análise de comportamento discriminatório.

A fim de verificar melhor o desempenho dos métodos Zbus e AIL com a variação da geração na barra 8, foram realizados alguns testes e os resultados são mostrados na Fig.2.

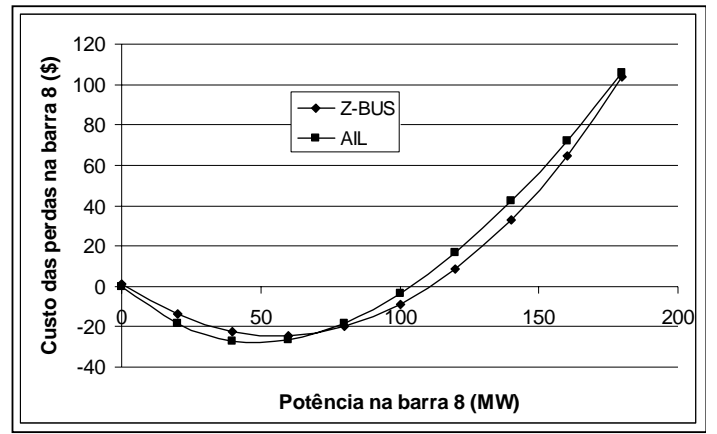

Figura 2. Custo das perdas alocados à barra 8 pelos métodos Zbus e AIL em função da variação de potência ativa.

Outros casos didáticos são simulados com o sistema REDE5, para analisar a questão relativa às alterações na rede (questão (f)). A seguir mostram-se algumas análises já realizadas em Expósito and Santos (2002), e Conejo et alii (2002a), e acrescentam-se resultados com outros métodos a fim de enfatizar aspectos positivos e negativos do método Z-bus. Os testes realizados são ilustrados na tabela 4.

Tabela 4 - Casos estudados para REDE5.

\begin{tabular}{ccc}
\hline Casos & Alterações na Rede & Custo Total \\
A1 & $\mathrm{T} 1=\mathrm{T} 2=1,0$ (caso base) & 1742,00 \\
A2 & $\mathrm{T} 1=1,1 \mathrm{e} \mathrm{T} 2=1,0$ & 2424,15 \\
$\mathrm{~A} 3$ & $\mathrm{~T} 1=\mathrm{T} 2=0,9$ & 1147,09 \\
$\mathrm{~A} 4$ & $\mathrm{~T} 1=\mathrm{T} 2=0,9 \mathrm{e}$ &
\end{tabular}

Capacitor de 100 Mvar na barra 1 1147,09
Tabela 5 - Alocação dos custos de perdas para o caso A1

\begin{tabular}{cccccccc|c}
\hline \multirow{2}{*}{ Barra } & \multicolumn{2}{l}{ PRO-RATA } & & \multicolumn{3}{c}{ ITL } & \\
& $(\mathrm{P})$ & $(\mathrm{I})$ & PS & ITL & $($ POS $)$ & AIL & Z-bus \\
$\mathrm{n}^{\circ}$ & $(\$)$ & $(\$)$ & $(\$)$ & $(\$)$ & $(\$)$ & $(\$)$ & $(\$)$ \\
1 & 420 & 745 & 390 & 359 & 871 & 369 & 338 \\
2 & 825 & 80 & 871 & 870 & 859,60 & 946 & 919 \\
3 & 469 & 917 & 481 & 512 & 11,40 & 426 & 484 \\
\hline
\end{tabular}

Tabela 6- Alocação dos custos de perdas para o caso A2.

\begin{tabular}{cccccccc}
\hline Barra & \multicolumn{2}{c}{ PRO-RATA } & & \multicolumn{5}{c}{ ITL } \\
& $(\mathrm{P})$ & $(\mathrm{I})$ & PS & ITL & $(\mathrm{POS})$ & AIL & Z-bus \\
$\mathrm{n}^{0}$ & $(\$)$ & $(\$)$ & $(\$)$ & $(\$)$ & $(\$)$ & $(\$)$ & $(\$)$ \\
1 & 600 & 859 & 502 & 519 & 1212 & 531 & 380 \\
2 & 1174 & 105 & 1210 & 1208 & 1133 & 1341 & 1217 \\
3 & 646 & 1456 & 709 & 696 & 78 & 578 & 824 \\
\hline
\end{tabular}

Tabela 7 - Alocação dos custos de perdas para o caso A3.

\begin{tabular}{ccccccccc}
\hline Barra & \multicolumn{2}{l}{ PRO-RATA } & & & ITL & & \\
& $(\mathrm{P})$ & $(\mathrm{I})$ & PS & ITL & (POS) & AIL & Z-bus \\
$\mathrm{n}^{\circ}$ & $(\$)$ & $(\$)$ & $(\$)$ & $(\$)$ & $(\$)$ & $(\$)$ & $(\$)$ \\
1 & 270 & 501 & 253 & 236 & 573 & 236 & 226 \\
2 & 565 & 472 & 573 & 573 & 569 & 625 & 602 \\
3 & 311 & 598 & 320 & 338 & 4,40 & 286 & 318 \\
\hline
\end{tabular}

Tabela 8 - Alocação dos custos de perdas para o caso A4.

\begin{tabular}{cccccccc|c}
\hline Barra & \multicolumn{2}{l}{ PRO-RATA } & \multicolumn{5}{c}{ ITL } \\
& $(\mathrm{P})$ & $(\mathrm{I})$ & PS & ITL & (POS) & AIL & Z-bus \\
& $\mathrm{n}^{\circ}$ & $(\$)$ & $(\$)$ & $(\$)$ & $(\$)$ & $(\$)$ & $(\$)$ & $(\$)$ \\
1 & 271 & 501 & 253 & 236 & 573 & 236 & 172 \\
2 & 565 & 472 & 574 & 573 & 569 & 625 & 715 \\
3 & 311 & 598 & 321 & 338 & 4,40 & 286 & 260 \\
\hline
\end{tabular}

Os sistemas IEEE14 e REDE5 são sistemas de pequeno porte e são bastante úteis para fins de verificar certas particularidades que indicam vantagens e desvantagens dos métodos. Para verificar o desempenho dos métodos frente a sistemas maiores passa-se agora a utilizar o sistema IEEE118. Nesta seção mostram-se os resultados obtidos com todos métodos de alocação de perdas apresentados anteriormente. Os resultados apresentados estão focados em duas regiões do sistema IEEE-118 barras, em torno da barra 12 e em torno da 67. A tabela 9 descreve os casos com seus respectivos custos que serão apresentados em detalhes nas tabelas de 10 à 14 . 
Tabela 9 - Casos estudados para Sistema IEEE118.

\begin{tabular}{ccc}
\hline Casos & Alterações na Rede & $\begin{array}{c}\text { Custo } \\
\text { Total }\end{array}$ \\
B1 & Caso base & 6637,88 \\
B2 & Gerador da barra 12 (de 85 MW para 95 MW) & 6590,14 \\
B3 & Gerador da barra 12 (de 85 MW para 75 MW) & 6688,83 \\
B4 & Carga da barra 67 (de 28 MW para 38 MW) & 6653,78 \\
B5 & Carga da barra 67 (de 28 MW para 18 MW) & 6623,88 \\
\hline
\end{tabular}

A tabela 10 mostra o custo de alocação para a rede original (caso base). Nota-se nesta tabela que o método Z-bus atribui incentivos monetários para a barra 12, ao invés de taxá-la alocando perdas. Isto como analisado anteriormente pode indicar que o gerador da barra 12 está localizado em um centro de consumo e para confirmar isto deve-se alterar a geração na barra 12 e verificar o que acontece com a soma de perdas em todas as ligações. Assim, apresentam-se na tabela 11 os resultados com aumento da geração na barra 12, e na tabela 12 com diminuição desta geração. Então se observa que o aumento de geração em 12 contribui para a diminuição das perdas no sistema, e a diminuição da geração em 12 contribui para o aumento de perdas. Este aumento de geração e diminuição de perdas traz benefícios para o sistema como um todo que poupa energia primária e também diminui custos alocados para a maioria das barras, portanto é razoável apresentar incentivos monetários para o gerador da barra 12, porém algumas barras têm seus custos aumentados, e isto pode ser visto como discriminatório. No caso didático com o IEEE14 este fato não apareceu. Agora a realocação de geração produziu uma redução no total de perdas, porém nem todas as barras receberam benefícios. Isto pode receber uma interpretação de alocação discriminatória, em que uma barra recebe incentivo monetário e outras são taxadas para "pagar” este incentivo.

Testes semelhantes aos realizados para indicar bom posicionamento de geradores podem ser feitos para determinar bom ou mal posicionamento de cargas. A tabela 12 mostra resultados dos custos alocados para as barras próximas à barra 67 e nas tabelas 13 e 14 com aumento e diminuição da carga em 67. Pode-se verificar que a barra 67 está bem posicionada no sistema, próxima ao centro de geração, e que aumento de carga na barra 67 provoca pequeno aumento de perdas no sistema (proporcionalmente muito baixo) e isto esta refletido na alocação realizada pelo método Z-bus.
Tabela 10. Custos alocados para barras com geração e barra 67 do sistema IEEE-118 para o caso B1.

\begin{tabular}{ccccccccc}
\hline Barra & \multicolumn{3}{|c}{ PRO-RATA } & & \multicolumn{3}{c}{ ITL } & \\
& $(\mathrm{P})$ & $(\mathrm{I})$ & PS & ITL & $($ POS $)$ & AIL & Z-bus \\
$\mathrm{n}^{\circ}$ & $(\$)$ & $(\$)$ & $(\$)$ & $(\$)$ & $(\$)$ & $(\$)$ & $(\$)$ \\
\hline 10 & 402 & 358 & 410 & 225 & 192 & 1319 & 210 \\
12 & 36 & 75 & 9 & 21 & 83 & 109 & -8 \\
25 & 196 & 178 & 284 & 138 & 213 & 389 & 185 \\
26 & 280 & 257 & 290 & 170 & 199 & 235 & 191 \\
49 & 104 & 117 & 11 & 91 & 169 & -78 & 21 \\
61 & 143 & 140 & 61 & 72 & 184 & -32 & 71 \\
65 & 349 & 336 & 238 & 253 & 217 & -214 & 335 \\
66 & 315 & 280 & 433 & 281 & 248 & 316 & 313 \\
67 & 25 & 24 & 9 & 9 & 25 & 32 & -14 \\
69 & 457 & 417 & 469 & 451 & 257 & 2528 & 627 \\
80 & 310 & 282 & 168 & 376 & 260 & -437 & 381 \\
87 & 4 & 10 & 1 & 5 & 314 & -4 & 1 \\
89 & 542 & 501 & 585 & 1184 & 438 & 0 & 2026 \\
100 & 192 & 186 & 223 & 183 & 249 & -303 & 187 \\
103 & 15 & 51 & 15 & 22 & 178 & -34 & -1 \\
111 & 32 & 31 & 19 & -10 & 59 & -94 & -36 \\
\hline
\end{tabular}

Tabela 11. Custos alocados para barras com geração e barra 67 do sistema IEEE-118 para o caso b2.

\begin{tabular}{|c|c|c|c|c|c|c|c|}
\hline \multirow{2}{*}{$\begin{array}{c}\text { Barra } \\
n^{0}\end{array}$} & \multicolumn{3}{|c|}{ PRO-RATA } & \multirow[b]{2}{*}{$\begin{array}{l}\text { ITL } \\
(\$)\end{array}$} & \multirow{2}{*}{$\begin{array}{c}\text { ITL } \\
\text { (POS) } \\
(\$)\end{array}$} & \multirow[b]{2}{*}{$\begin{array}{l}\text { AIL } \\
(\$)\end{array}$} & \multirow[b]{2}{*}{$\begin{array}{c}\text { Z-bus } \\
\text { (\$) }\end{array}$} \\
\hline & $\begin{array}{l}(\mathrm{P}) \\
(\$)\end{array}$ & $\begin{array}{c}(\mathrm{I}) \\
(\$)\end{array}$ & $\begin{array}{l}\text { PS } \\
(\$)\end{array}$ & & & & \\
\hline 10 & 399 & 356 & 408 & 244 & 200 & 1320 & 243 \\
\hline 12 & 43 & 76 & 11 & 21 & 85 & 132 & -17 \\
\hline 25 & 195 & 177 & 282 & 143 & 219 & 389 & 193 \\
\hline 26 & 279 & 255 & 290 & 178 & 204 & 235 & 203 \\
\hline 49 & 104 & 117 & 110 & 90 & 165 & -78 & 18 \\
\hline 61 & 142 & 139 & 61 & 71 & 182 & -32 & 65 \\
\hline 65 & 347 & 334 & 234 & 249 & 216 & -212 & 323 \\
\hline 66 & 313 & 279 & 431 & 277 & 248 & 316 & 303 \\
\hline 67 & 25 & 23 & 9 & 9 & 25 & 32 & -14 \\
\hline 69 & 446 & 408 & 459 & 434 & 256 & 2469 & 592 \\
\hline 80 & 308 & 281 & 168 & 370 & 259 & -434 & 366 \\
\hline 87 & 4 & 10 & 1 & 5 & 316 & -3 & 0 \\
\hline 89 & 539 & 498 & 585 & 1172 & 446 & 4 & 1997 \\
\hline 100 & 191 & 185 & 223 & 179 & 247 & -302 & 177 \\
\hline 103 & 15 & 51 & 15 & 22 & 173 & -34 & -2 \\
\hline 111 & 32 & 30 & 19 & -11 & 49 & -94 & -38 \\
\hline
\end{tabular}


Tabela 12. Custos alocados para barras com geração e barra 67 do sistema IEEE-118 para o caso b3.

\begin{tabular}{|c|c|c|c|c|c|c|c|}
\hline \multirow{2}{*}{$\begin{array}{c}\text { Barra } \\
n^{\circ}\end{array}$} & \multicolumn{2}{|c|}{ PRO-RATA } & \multirow[b]{2}{*}{$\begin{array}{l}\text { PS } \\
(\$)\end{array}$} & \multirow[b]{2}{*}{$\begin{array}{l}\text { ITL } \\
(\$)\end{array}$} & \multirow{2}{*}{$\begin{array}{c}\text { ITL } \\
\text { (POS) } \\
(\$)\end{array}$} & \multirow[b]{2}{*}{$\begin{array}{l}\text { AIL } \\
\text { (\$) }\end{array}$} & \multirow[b]{2}{*}{$\begin{array}{c}\text { Z-bus } \\
\text { (\$) }\end{array}$} \\
\hline & $\begin{array}{l}(\mathrm{P}) \\
(\$)\end{array}$ & $\begin{array}{l}(\mathrm{I}) \\
(\$)\end{array}$ & & & & & \\
\hline 10 & 405 & 360 & 414 & 195 & 182 & 1318 & 160 \\
\hline 12 & 25 & 74 & 6 & 24 & 81 & 74 & 9 \\
\hline 25 & 198 & 180 & 287 & 129 & 206 & 389 & 174 \\
\hline 26 & 283 & 259 & 291 & 157 & 192 & 235 & 173 \\
\hline 49 & 105 & 119 & 113 & 93 & 175 & -79 & 25 \\
\hline 61 & 144 & 141 & 62 & 75 & 187 & -33 & 79 \\
\hline 65 & 352 & 339 & 245 & 258 & 218 & -216 & 352 \\
\hline 66 & 318 & 282 & 437 & 286 & 248 & 315 & 330 \\
\hline 67 & 25 & 24 & 9 & 9 & 25 & 32 & -16 \\
\hline 69 & 473 & 431 & 485 & 476 & 257 & 2621 & 680 \\
\hline 80 & 313 & 285 & 167 & 386 & 261 & -441 & 402 \\
\hline 87 & 4 & 10 & 1 & 5 & 311 & -4 & 1 \\
\hline 89 & 547 & 504 & 585 & 1201 & 428 & -6 & 2071 \\
\hline 100 & 194 & 188 & 223 & 189 & 251 & -306 & 202 \\
\hline 103 & 15 & 51 & 15 & 23 & 185 & -34 & -1 \\
\hline 111 & 32 & 31 & 19 & -9 & 72 & -95 & -33 \\
\hline
\end{tabular}

Tabela 13. Custos alocados para barras com geração do e barra 67 sistema IEEE-118 para o caso b4.

\begin{tabular}{|c|c|c|c|c|c|c|c|}
\hline \multirow{2}{*}{$\begin{array}{c}\text { Barra } \\
n^{0}\end{array}$} & \multicolumn{3}{|c|}{ PRO-RATA } & \multirow[b]{2}{*}{$\begin{array}{l}\text { ITL } \\
(\$)\end{array}$} & \multirow{2}{*}{$\begin{array}{c}\text { ITL } \\
\text { (POS) } \\
(\$)\end{array}$} & \multirow[b]{2}{*}{$\begin{array}{l}\text { AIL } \\
(\$)\end{array}$} & \multirow[b]{2}{*}{$\begin{array}{c}\text { Z-bus } \\
\text { (\$) }\end{array}$} \\
\hline & $\begin{array}{l}(\mathrm{P}) \\
(\$)\end{array}$ & $\begin{array}{l}(\mathrm{I}) \\
(\$)\end{array}$ & $\begin{array}{l}\text { PS } \\
(\$)\end{array}$ & & & & \\
\hline 10 & 402 & 357 & 410 & 224 & 192 & 1314 & 209 \\
\hline 12 & 36 & 75 & 9 & 21 & 83 & 108 & -8 \\
\hline 25 & 196 & 178 & 284 & 138 & 214 & 386 & 186 \\
\hline 26 & 280 & 257 & 291 & 169 & 199 & 235 & 191 \\
\hline 49 & 104 & 117 & 111 & 90 & 169 & -79 & 20 \\
\hline 61 & 143 & 140 & 60 & 71 & 183 & -32 & 69 \\
\hline 65 & 349 & 336 & 245 & 252 & 217 & -216 & 333 \\
\hline 66 & 315 & 280 & 427 & 279 & 248 & 315 & 311 \\
\hline 67 & 34 & 31 & 13 & 13 & 25 & 44 & -17 \\
\hline 69 & 466 & 425 & 479 & 464 & 259 & 2574 & 648 \\
\hline 80 & 310 & 282 & 166 & 377 & 261 & -441 & 382 \\
\hline 87 & 4 & 10 & 1 & 5 & 315 & -4 & 1 \\
\hline 89 & 542 & 501 & 585 & 1185 & 439 & -17 & 2029 \\
\hline 100 & 192 & 186 & 223 & 183 & 250 & -305 & 188 \\
\hline 103 & 15 & 51 & 15 & 22 & 179 & -34 & -1 \\
\hline 111 & 32 & 31 & 19 & -10 & 60 & -94 & -36 \\
\hline
\end{tabular}

Tabela 14. Custos alocados para barras com geração e barra 67 do sistema IEEE-118 para o caso b5.

\begin{tabular}{|c|c|c|c|c|c|c|c|}
\hline Barra & $\begin{array}{c}\text { PRO- } \\
(\mathrm{P}) \\
(\$)\end{array}$ & $\begin{array}{l}\text { ATA } \\
\text { ( I ) } \\
\text { (\$) }\end{array}$ & $\begin{array}{l}\text { PS } \\
(\$)\end{array}$ & $\begin{array}{l}\text { ITL } \\
(\$)\end{array}$ & $\begin{array}{c}\text { ITL } \\
\text { (POS) } \\
(\$)\end{array}$ & $\begin{array}{l}\text { AIL } \\
(\$)\end{array}$ & $\begin{array}{c}\text { Z-bus } \\
\text { (\$) }\end{array}$ \\
\hline 10 & 36 & 75 & 9 & 22 & 83 & 109 & -8 \\
\hline 12 & 197 & 178 & 284 & 138 & 213 & 392 & 185 \\
\hline 25 & 281 & 257 & 290 & 170 & 198 & 235 & 191 \\
\hline 26 & 105 & 117 & 111 & 92 & 169 & -77 & 21 \\
\hline 49 & 143 & 140 & 63 & 74 & 185 & -32 & 72 \\
\hline 61 & 349 & 336 & 232 & 254 & 217 & -211 & 336 \\
\hline 65 & 315 & 280 & 441 & 283 & 248 & 316 & 316 \\
\hline 66 & 16 & 16 & 5 & 5 & 24 & 20 & -11 \\
\hline 67 & 448 & 409 & 459 & 438 & 255 & 2484 & 606 \\
\hline 69 & 310 & 282 & 169 & 376 & 259 & -433 & 379 \\
\hline 80 & 4 & 10 & 1 & 5 & 312 & -3 & 1 \\
\hline 87 & 542 & 501 & 585 & 1183 & 437 & 17 & 2024 \\
\hline 89 & 192 & 186 & 223 & 183 & 248 & -301 & 186 \\
\hline 100 & 15 & 51 & 15 & 22 & 177 & -34 & -1 \\
\hline 103 & 32 & 31 & 19 & -10 & 58 & -94 & -36 \\
\hline 111 & 36 & 75 & 9 & 22 & 83 & 109 & -8 \\
\hline
\end{tabular}

\section{CONCLUSÕES}

Este trabalho apresentou as principais técnicas para alocação de perdas utilizadas atualmente em redes de transmissão, e realizou uma análise crítica. Todos os métodos não apresentam problemas para entendimento e implementação. Os métodos pro-rata P e I não dependem da rede e isto pode representar uma desvantagem. O métodos ITL, AIL e Z-bus apresentam alocação positiva ou negativa, sendo que o método Z-bus apresenta alocação negativa com maior freqüência. $\mathrm{O}$ fato de apresentarem alocação negativa é uma vantagem. A alocação negativa, realizada pelos métodos AIL, ITL e Z-bus, informa quais barras (cargas e geradores) são bem posicionados na rede, e isto pode servir de indicativo para acréscimo de geração ou de carga, em determinadas regiões. Obviamente esta indicação de bons locais para novos geradores (ou cargas) deve ser tomada com avaliação de alocação de perdas para uma curva de carga diária (24 horas).

Um problema importante apresentado pela maioria dos métodos é realizar alocação de forma discriminatória. Os métodos que fazem alocação negativa, muitas vezes, são os que mais apresentam a alocação discriminatória, pois as barras que recebem alocação positiva pagam as perdas 
globais e mais os incentivos (alocação negativa). A maioria das publicações da área tem mostrado o desempenho das propostas utilizando sistemas elétricos de pequeno porte, onde o comportamento discriminatória aparece pouco ou não aparece. Neste trabalho foram obtidos resultados com sistemas pequenos e também com o IEE118. Maiores investigações são necessárias visando uma melhor maneria de considerar a alocação discriminatória, focando não somente as perdas mas também uma avaliação de fluxos na rede.

As análises realizadas mostram que fazer a alocação de perdas utilizando apenas um método pode não ser uma boa solução para todos os geradores e cargas, pois alocação discriminatória pode aparecer. Um bom indicativo de procedimento é trabalhar com mais de um método para alocar os custos. Recomenda-se utilizar pelo menos um método que faça alocação negativa, a fim de obter informações sobre barras bem posicionadas. Na atribuição de custos com mais de um procedimento, um método pode ser utilizado para alocar as perdas e outro para produzir incentivos de forma a corrigir alocações discriminatórias. Porém estas análises necessitam de mais investigações.

\section{AGRADECIMENTOS}

Os autores agradecem a Fapesp, projeto número 00/119048, pelo apoio financeiro. Agradecem também a UNESP e a CTEEP.

\section{REFERÊNCIAS BIBLIOGRÁFICAS}

Bialek, J. (1996). Tracing the flow of electricity. IEEE Proc-Gener. Transm. Distrib, 143 (4), pp. 313-320.

Conejo, A. J., Galiana, F.D. and Kockar, I (2001). Z-Bus loss allocation. IEEE Trans. on Power Systems,16 (1), pp. 105-110.

Conejo, A.J. Galiana, F.D. and Kockar, I. (2002a). Closure to discussion of “Z-bus loss allocation”. IEEE Trans. On Power Systems, 17 (2), pp. 526-527.

Elgerd,O.I (1982). Electric Energy Systems Theory: An Introduction. McGraw-Hill , New York.

Expósito, A.G., Santos, J.M.R., Garcia, T.G. and Velascos E.A.R. (2000). Fair allocation of transmission power losses. IEEE Trans. on Power Systems, 15 (1), pp. 184188.
Expósito, A.G. and Santos, J.R. (2002) Discussion of “Zbus loss allocation”. IEEE Trans. On Power Systems, 17 (2), pp. 525.

Fernandes, T.S.P. and Almeida, K.C. (2002). Methodologies for loss and line flow allocation under a pool-bilateral market. Proc. of $14^{\text {th }}$ PSCC, Session 23, Paper2, pp-1-7.

Galiana, F.D., Conejo, A.J. and Kockar, I. (2002). Incremental transmission loss allocation in a transaction framework. IEEE Transactions on Power Systems, 17 (1), pp:26-33.

Gonzalez, J.J. and Basagoiti, P. (1999). Spanish power exchange market and information system. Design concepts, and operating experience. In: Proceeding of IEEE Power Industry Computer Applications Conference, pp. 245-252.

Glover, J.D and Sarma, M. (1987). Power System Analysis and Design. Kluver Academic Publishers, Boston, MA.

Leite da Silva, A M., Costa, J.G.C, Mello, J.C.O, Abreu, J.C., Romero,S.P., Treistman,R. (2001). Determinação dos fatores de perdas aplicados na medição do mercado atacadista de energia elétrica. In: XVI SNPTEE, artigo GAT-018, pp. 1-5.

Leite da Silva, A M. and Costa, J.G.C. (2003). Transmission loss allocation: Part I - single energy market. IEEE Trans. on Power Systems, 18 (4), pp. 1389-1394.

Schweppe, F., Caramanis M., Tabors R. and Bohn R.(1988). Spot Pricing of Electricity, Kluver Academic Publishers, Boston, MA.

The Standard IEEE 118 test data (2003). http//:www.ee.washington.edu/research/pstca/sensitivit y/sensreadme.txt. Disponível em Janeiro de 2003. 


\section{APÊNDICE A}

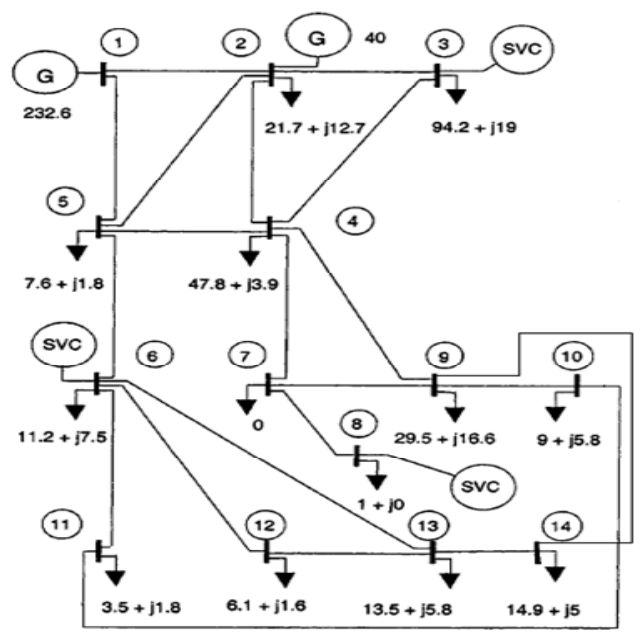

Figura A1. Rede IEEE 14 barras.

Tabela A1. Dados de linhas IEEE 14 barras.

\begin{tabular}{cccccc}
\hline linha $\mathrm{n}^{\mathrm{o}}$ & $\begin{array}{c}\text { Barra } \\
\text { origem }\end{array}$ & $\begin{array}{c}\text { Barra } \\
\text { destino }\end{array}$ & $\mathrm{r}(\mathrm{pu})$ & $\mathrm{x}(\mathrm{pu})$ & $\mathrm{B}(\mathrm{pu})$ \\
1 & 1 & 2 & 0,019 & 0,059 & 0,053 \\
2 & 1 & 5 & 0,054 & 0,223 & 0,053 \\
3 & 2 & 3 & 0,047 & 0,198 & 0,044 \\
4 & 2 & 4 & 0,058 & 0,176 & 0,037 \\
5 & 2 & 5 & 0,057 & 0,174 & 0,034 \\
6 & 3 & 4 & 0,067 & 0,171 & 0,035 \\
7 & 4 & 5 & 0,013 & 0,042 & 0,013 \\
8 & 4 & 7 & 0,000 & 0,209 & 0,000 \\
9 & 4 & 9 & 0,000 & 0,556 & 0,000 \\
10 & 4 & 6 & 0,000 & 0,252 & 0,000 \\
11 & 6 & 11 & 0,095 & 0,199 & 0,000 \\
12 & 6 & 12 & 0,123 & 0,256 & 0,000 \\
13 & 6 & 13 & 0,066 & 0,130 & 0,000 \\
14 & 7 & 8 & 0,000 & 0,176 & 0,000 \\
15 & 7 & 9 & 0,000 & 0,110 & 0,000 \\
16 & 9 & 10 & 0,032 & 0,085 & 0,000 \\
17 & 9 & 14 & 0,127 & 0,270 & 0,000 \\
18 & 10 & 11 & 0,082 & 0,192 & 0,000 \\
19 & 12 & 13 & 0,221 & 0,200 & 0,000 \\
20 & 13 & 14 & 0,171 & 0,348 & 0,000 \\
\hline & & & & &
\end{tabular}

\section{APÊNDICE B}

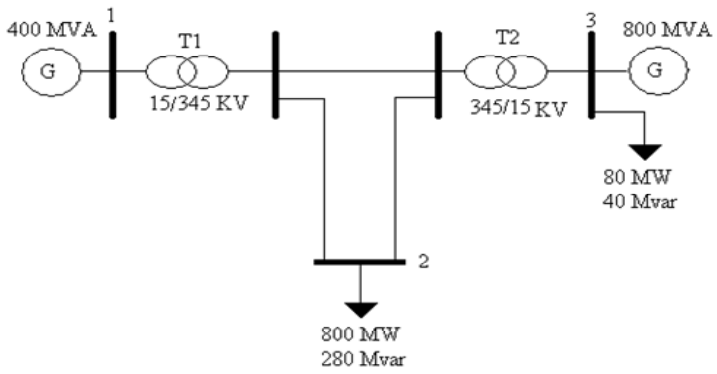

Figura B1. Rede 5 barras

Tabela B2. Dados de barras da Rede 5.

\begin{tabular}{ccccc}
\hline Barra & PG (MW) & QG (MVAR) & PL (MW) & QL (MW) \\
1 & - & - & - & - \\
2 & 0 & 0 & 800 & 280 \\
3 & 520 & - & 80 & 40 \\
4 & 0 & 0 & 0 & 0 \\
5 & 0 & 0 & 0 & 0 \\
\hline
\end{tabular}

Tabela B2. Dados de barras da Rede 5.

\begin{tabular}{ccccccc}
\hline linha $\mathrm{n}^{\circ}$ & $\begin{array}{c}\text { Barra } \\
\text { origem }\end{array}$ & $\begin{array}{c}\text { Barra } \\
\text { destino }\end{array}$ & $\mathrm{r}(\mathrm{pu})$ & $\mathrm{x}(\mathrm{pu})$ & $\mathrm{B}(\mathrm{pu})$ & Transf.. \\
1 & 1 & 5 & 0,006 & 0,0800 & 0,0 & 1,0 \\
2 & 2 & 4 & 0,0360 & 0,4000 & 0,4300 & - \\
3 & 2 & 5 & 0.0180 & 0.200 & 0.2200 & - \\
4 & 4 & 5 & 0,0090 & 0,100 & 0,1100 & - \\
5 & 3 & 4 & 0,0030 & 0,0400 & 0,00 & 1,0 \\
\hline
\end{tabular}



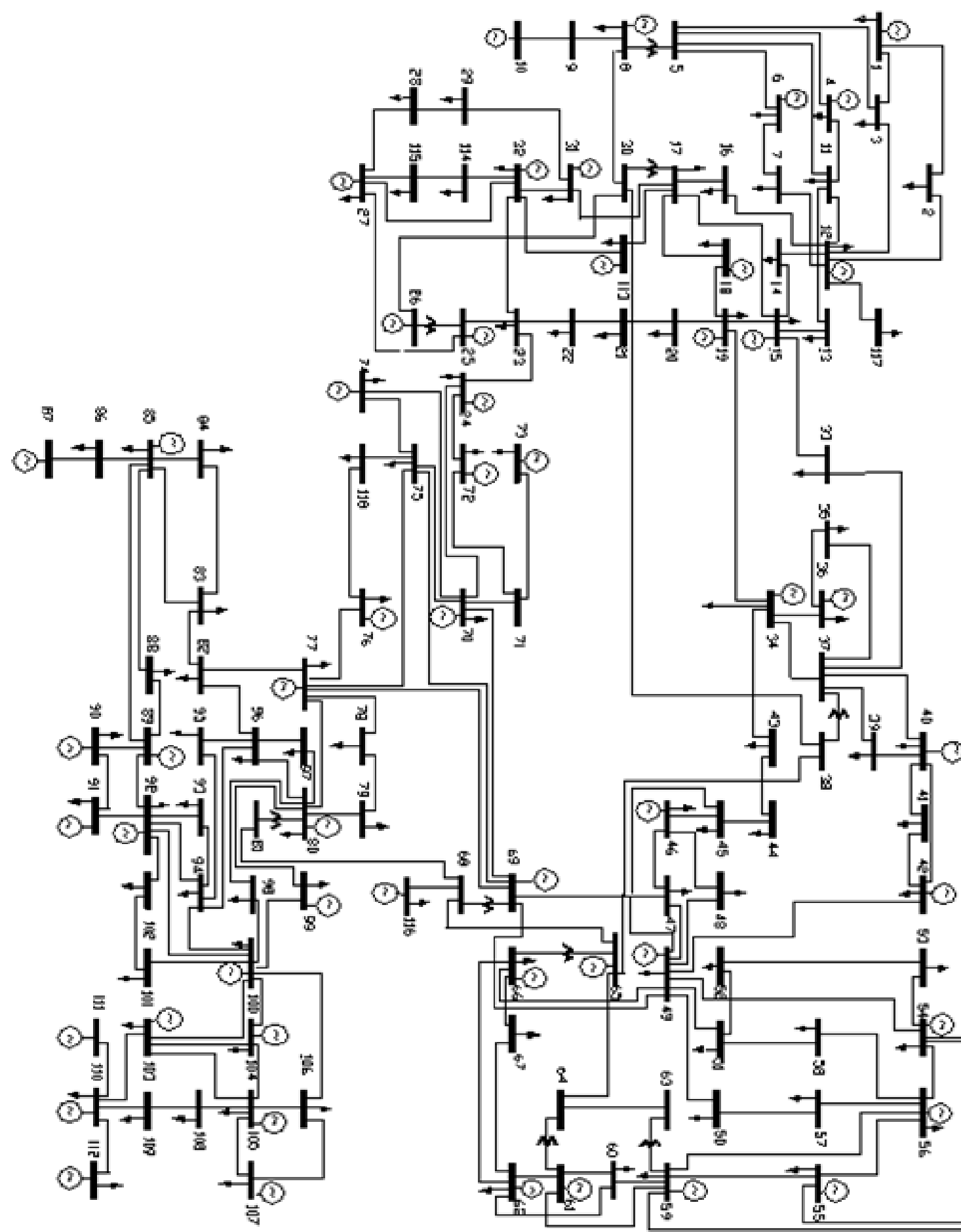